\title{
TV/Series
}

18 | 2020

Séries et espace

\section{Atlanta (FX, Donald Glover, 2016-), vers une transgression générique des frontières géographiques et sociales}

\section{Yvelin Ducotey}

\section{OpenEdition}

Journals

Édition électronique

URL : http://journals.openedition.org/tvseries/4571

DOI : $10.4000 /$ tvseries.4571

ISSN : 2266-0909

Éditeur

GRIC - Groupe de recherche Identités et Cultures

Référence électronique

Yvelin Ducotey, "Atlanta (FX, Donald Glover, 2016-), vers une transgression générique des frontières géographiques et sociales », TV/Series [En ligne], 18| 2020, mis en ligne le 15 septembre 2020, consulté le 08 décembre 2020. URL : http://journals.openedition.org/tvseries/4571 ; DOI : https:// doi.org/10.4000/tvseries.4571

Ce document a été généré automatiquement le 8 décembre 2020.

\section{(i) $९$

TV/Series est mis à disposition selon les termes de la licence Creative Commons Attribution - Pas d'Utilisation Commerciale - Pas de Modification 4.0 International 


\title{
Atlanta (FX, Donald Glover, 2016-), vers une transgression générique des frontières géographiques et sociales
}

\author{
Yvelin Ducotey
}

1 Atlanta s'inscrit dans la lignée de séries qui situent leurs propos dans un cadre spatial particulier. Ici, l'espace urbain et banlieusard américain d'Atlanta (Géorgie) constitue un acteur majeur de la trame narrative. Comme le fait remarquer Georges Molinié, « il existe intrinsèquement un rapport inhérent entre l'espace et le pictural, entre l'espace et le photographique, entre l'espace et le cinématographique ${ }^{1} »$. Donald Glover a pleinement conscience de l'importance d'un tel rapport qu'Atlanta explore en détail, notamment d'un point de vue sociologique, racial et, par intermittence, genré.

2 Il n'est pas rare que les séries qui s'inscrivent dans l'espace urbain s'intéressent particulièrement aux marges et périphéries, deux termes primordiaux dans l'analyse d'Atlanta. Un exemple serait The Wire (HBO, David Simon, 2002-2008), qui a cartographié une partie de la ville de Baltimore (Maryland). David Simon, créateur et auteur de la série, s'est attaché à dépeindre avec attention le monde de la criminalité dans un espace urbain fortement impacté par la violence, notamment sociale ${ }^{2}$. Dans un autre genre, citons Desperate Housewives (ABC, Marc Cherry, 2004-2012) et sa banlieue pavillonnaire dont le vernis de la perfection se craquelle d'épisode en épisode.

3 Le spectateur navigue dans un univers restreint et son exploration, de plus en plus immersive, lui permet de découvrir plus en détail ce monde et d'en apprendre les moindres secrets. Pour autant, l'étude du micro (un nombre de rues limité) se veut représentative d'une attention macroscopique, reflet de certaines réalités sociales à un instant précis. Atlanta ne départit pas d'une telle volonté. Donald Glover, à travers le portrait de ses personnages, propose de mener une réflexion sur la situation de la jeunesse afro-américaine qui évolue dans ces espaces urbains limités et marginaux, un terme qui sera développé dans toute sa polysémie (marginalité spatiale, mais aussi 
sociale et raciale). Par ce premier aspect, Atlanta se rapproche du genre de la critique sociale au cinéma qui, selon Franck Fischbach, « commence avec le fait de s'apercevoir que quelque chose ne va pas, que quelque chose cloche dans nos rapports sociaux, que, dans notre vie sociale, les choses ne vont effectivement pas comme elles devraient aller » [en italique dans le texte] ${ }^{3}$.

4 La mise en scène de diverses zones frontière de conflit que nous allons évoquer témoigne du fait que «quelque chose ne va pas» dans les rapports sociaux et urbains à Atlanta.

Nombreuses sont donc les séries qui visent à imbriquer l'évolution de leurs personnages dans un contexte social particulier, constitutif et étroitement lié à l'espace mis en scène. Le phénomène effleuré ici n'est pas propre à la série, et semble même être hérité de la culture cinématographique ${ }^{4}$. Cependant, notre article a pour ambition de souligner en quoi le format sériel est idoine pour une exploration détaillée et sociologique d'un espace urbain particulier.

6 Comme de nombreux films et séries, Atlanta s'intéresse à des populations marginalisées. Les deux saisons prises en compte pour le présent article suivent le parcours d'Earl (Donald Glover), jeune homme africain-américain qui se démène pour vivre ses rêves tout en tâchant de subvenir aux besoins de sa fille. Une des spécificités de la série est de mettre en avant, uniquement ou presque, des personnages noirs, issus des classes sociales les plus défavorisées: Van (Zazie Beetz), le rappeur Paper Boi ${ }^{5}$ (Bryan Tyree Henry), et son acolyte Darius (Lakeith Stanfield), junkie et philosophe moderne, dont la lucidité s'avère parfois déroutante.

7 Avec la série Atlanta, Donald Glover cherche initialement à cartographier la ville éponyme, mettant en scène des frontières sociétales a priori infranchissables. Dans le cadre de cet article, le terme de "ville» doit être compris comme "territoire et population, cadre matériel et unité de vie collective, configuration d'objets physiques et nœud de relations entre sujets sociaux ${ }^{6}{ }$. Donald Glover imbrique le territoire et ses populations, témoignant de la réalité quotidienne de ses personnages. L'important est de mettre en lumière la manière avec laquelle le créateur de la série inscrit ses protagonistes dans un espace limité qui influence leur vie journalière. Un effort notable dès le pilote qui permet une contextualisation sociale des propos de la série.

Dans Atlanta, la relation au territoire urbain se développe en trois temps, chacun étant lié à une géographie particulière correspondant aux différentes parties du présent article. Dans un premier temps, Glover semble avoir une conception antinomique de l'espace, mettant en scène une aire étouffante et imposée aux protagonistes, et de laquelle ils ne parviennent pas à s'extraire. Mais comme l'expriment David Apter et Philippe de Lavergne, «le paysage a beau être dégagé et le terrain visible, l'espace cache beaucoup plus de choses qu'il ne révèle ${ }^{7}$ ». En effet, et dans un deuxième temps, au-delà de l'emprisonnement perceptible dès le premier épisode de la série, Glover met en images diverses zones de tension, entre différents groupes sociaux et raciaux. Elles dévoilent de nombreuses frontières qui participent à une délimitation franche de multiples enclaves urbaines. Dans un dernier temps, et notamment au cours de la saison 2, Atlanta évoque les stratégies, tant intra qu'extradiégétiques, employées par le créateur de la série pour s'extraire d'un cadre spatial restreint et imposé. 


\section{Espace diégétique restreint et répercussions sociales}

9 Dès le premier épisode, Donald Glover entre dans le vif du sujet en mettant en scène un sentiment de cloisonnement spatial intrinsèque à la situation sociale de ses personnages. La séquence introductive n'a d'ouverture que son nom. Loin du centreville d'Atlanta, la série débute en périphérie, de nuit, sur le parking d'une supérette. La contextualisation territoriale justifie le choix de la ville d'Atlanta comme toile de fond pour la série. Appartenant au Sud étatsunien, la ville a vu le nombre de banlieues augmenter progressivement au cours des luttes pour les droits civiques à partir des années $1960^{8}$. Pour autant, la géographie urbaine est révélatrice des inégalités sociales qui font encore rage. En 1996, la population noire représente les trois quarts des habitants de la ville, mais elle se voit obligée d'évoluer dans les couronnes suburbaines, un positionnement révélateur de «l'élargissement du fossé entre les groupes sociaux ${ }^{9} »$.

10 Apparaissent alors Paper Boi, Darius et Earn, filmés dans un plan serré à l'intérieur même de la voiture, le corps massif de Paper Boi étant comme entravé, comme prisonnier de sa propre condition. L'identité du premier est très vite révélée : il est un rappeur au succès foudroyant après la sortie de son titre éponyme. L'univers du rap, et plus exactement $d u$ "trap» (courant originaire d'Atlanta ${ }^{10}$ ), sera la toile de fond musicale de la série. Glover précise immédiatement le statut social de ses protagonistes dont le quotidien semble régi par la pauvreté et la violence, les deux étant intrinsèquement liées. Par cette contextualisation socio-spatiale, Donald Glover détermine l'attachement forcé qui unit ses personnages aux lieux qu'ils occupent. Alors qu'Earn et son cousin décident de se rendre en «boite ", avant 22h45, afin d'obtenir une réduction, une altercation éclate avec un autre client des premières heures du club. La violence va crescendo, jusqu'à la sortie de deux armes, le pré-générique se clôturant sur un bruit de détonation sans que le spectateur ne puisse voir ce qu'il se passe.

11 La première séquence fige les personnages dans un environnement urbain clos, mais aussi dans un rôle précis et attendu. Donald Glover joue avec les archétypes liés au monde de la musique rap. Son cousin, Paper Boi, porte un t-shirt trop large, un short baggy, ainsi qu'une chaîne en or disproportionnée illustrant son succès soudain. Quant à son nom, il fait explicitement allusion à l'importance donnée à l'argent ${ }^{11}$. À cela s'ajoute la violence des armes à feu, autre élément archétypal de la représentation de la culture rap (et auquel renvoie le titre de l'épisode: The Big Bang), ainsi que la drogue. Glover pare initialement ses protagonistes d'un « costume social» en adéquation avec l'espace filmé. Paper Boi et Darius sont des personnages types, répondant à « l'horizon d'attentes" jaussien, propre à cet univers précis, notamment en raison de "l'expérience que le public a du genre dont elle [la série] relève ${ }^{12}$ ». Le sentiment instantané d'immobilité tient aussi à la mise en scène. La caméra n'est jamais en mouvement et, bien que portée à l'épaule, aucun travelling ni panoramique n'est perceptible.

12 Le dernier plan de la séquence influe considérablement sur la lecture de cette introduction. La détonation qui ponctue la scène est filmée dans une plongée verticale polysémique. Un tel choix de cadrage implique un certain déterminisme (social et spatial) qui dépasse les protagonistes. Elle précise la position des personnages, au bas de l'échelle sociale ; ils sont comme enchaînés, sans possibilité aucune de s'élever. Par l'absence de ligne de fuite, la verticalité obstrue l'horizon et les prive de liberté de 
mouvement. La plongée zénithale laisse ainsi entendre qu'ils sont le fruit d'un environnement spécifique.

13 Le choix de mise en scène de Hiro Murai est renforcé par la circularité de la construction narrative qui confirme l'absence de perspectives dont souffrent les personnages. Le pré-générique est une sombre prévision puisque le reste de l'épisode se déroule avant cette séquence. Il faut attendre le deuxième épisode ("Streets on Locks ", titre renvoyant également au sentiment d'enfermement) pour découvrir les conséquences de l'altercation. Earn et son cousin apparaissent alors au commissariat. La construction circulaire du scénario sonne telle une prédiction à laquelle il sera difficile de se soustraire. Toute la question est de savoir si Earn, Paper Boi et Darius vont pouvoir s'arracher de leur environnement en dépassant les frontières - tant géographiques que sociales - établies dès le premier épisode, et par quel moyen ? Le sentiment de déterminisme socio-spatial renvoie indéniablement à Menace II Society (Albert et Allen Hughes, 1993), récit d'une descente aux enfers inexorable pour le personnage de Caine (Tyrin Turner), jeune africain-américain. Le film se déroule dans les quartiers périphériques de Los Angeles, un an après les émeutes de 1992 et le meurtre de Rodney King ${ }^{13}$, confirmant l'importance de l'espace urbain dans l'évolution des personnages.

14 À l'issue de la première scène, Darius parachève le sentiment de déterminisme social. Alors que l'altercation susmentionnée s'envenime, Darius a « un sentiment de déjà$v u$ », signifiant ainsi la fréquence élevée avec laquelle ce genre d'incidents surviennent dans ces espaces urbains périphériques. Atlanta est l'une des villes américaines les plus frappées par la violence (51 meurtres pour 100000 habitants, troisième plus haut taux en 1996$)^{14}$. Finalement, la toute première scène d'Atlanta reflète la dureté quotidienne qui affecte les résidents africains-américains et démunis de cette partie de la ville.

Atlanta se caractérise donc par la circularité de sa trame narrative, sous-entendant que, malgré tous leurs efforts, les personnages ne parviendront jamais à aller de l'avant. Mais le choix scénaristique de Donald Glover se veut également le reflet du modèle urbain concentrique développé par le sociologue Ernest Burgess ${ }^{15}$. Selon sa théorie, "les citadins se distribuent en zones relativement typées, depuis le centre des affaires jusqu'aux lointaines couronnes suburbaines ${ }^{16} »$. Par son introduction, la série Atlanta présente une de ces "zones typées", tandis que les personnages occupent une " couronne suburbaine", sans parvenir à atteindre un cercle plus proche du point central de la ville. La construction non linéaire de la série laisse entendre qu'Atlanta, par la voix de son créateur, exprime une critique sociale. Comme le fait remarquer Franck Fischbach :

La circularité du film [...] constitue justement l'une des figures cinématographiques de la critique sociale dans la mesure où cette circularité permet d'exprimer l'idée d'un monde sociale qui soit un enfermement, une "cage d'acier" comme disait Max Weber. ${ }^{17}$

16 Nombreux seront les effets de mise en scène qui tendent à décrire l'environnement des personnages comme une cage d'acier, notamment l'apparition des protagonistes dans leur voiture.

17 Le générique renforce le sentiment d'une ville socialement quadrillée et délimitée par diverses frontières. Hiro Murai, réalisateur du premier épisode, juxtapose un enchaînement de plongées zénithales mettant en scène les différentes réalités sociales disparates d'Atlanta. Aux plans de quartiers défavorisés, succèdent des images de riches 
villas, attestant que «la diversité des types d'habitats et leur répartition dans la ville sont les marques les plus visibles de la séparation des groupes sociaux ${ }^{18} »$. La juxtaposition spatiale se prolonge par le format sériel d'Atlanta qui propose aux spectateurs une succession d'enclaves narratives. Le réalisateur met également en exergue l'impossible communication entre ces groupes sociaux, "la clôture est montrée comme telle grâce à la vision aérienne ${ }^{19}$. » Aucun fondu enchaîné ne crée de lien, au contraire, les coupes franches imposent une frontière qui établit une norme centrale et une périphérie circulaire. Aucun carrefour, tant spatial que social, n'est perceptible dans cette cartographie de la ville. La succession des plans aériens répond alors à un modèle "nodulaire", "selon lequel l'espace de la ville est constitué de noyaux de populations plus ou moins spécifiques qui peuvent constituer, dans les cas extrêmes, des "enclaves" fortement typées du point de vue de l'appartenance sociale $^{20} »$. La description archétypale de Paper Boi et Earn lors de leur entrée dans le cadre favorise la lecture de l'espace comme une " enclave fortement typée ».

La mise en scène confère à la série et à ses différentes aires urbaines un esprit communautaire fort, relayé par les dialogues, eux aussi révélateurs. La bande-originale de la série, qui n'est pas seulement propre à la ville d'Atlanta, mais plus encore, aux quartiers mis en scènes, joue un rôle similaire. Les personnages multiplient l'usage du "nous ", marquant ainsi une frontière verbale et communicationnelle entre « eux et nous » pour reprendre le schéma développé par Richard Hoggart sur les cultures populaires ${ }^{21}$. Au-delà de l'appropriation de l'espace par un groupe particulier, «l'espace de la ville est aussi un enjeu de compétitions, voire de "luttes" pour son appropriation matérielle et symbolique ${ }^{22} »$. Les nombreuses oppositions entre divers groupes sociaux qui rythment la série reflètent l'esprit de compétition dont font mention Grafmeyer et Authier, mais surtout, confirment l'impossible communication pressentie dans le générique inaugural. La ville d'Atlanta, telle que dépeinte, expose donc l'espace urbain " en tant que lieu d'appartenance», tandis que la ville "englobe les expériences sociales, politiques et raciales qui façonnent l'identité des communautés qui y sont représentées ${ }^{23} »$. Les enjeux urbains s'articulent autour d'une volonté d'appropriation de l'espace par certains groupes étanches, car la ville demeure « intrinsèquement liée au pouvoir ${ }^{24}$ ", le cœur de ville étant l'épicentre de ce pouvoir. En somme, « le droit à la ville est aussi un droit de prise de position sur la ville ${ }^{25} »$.

La répétition des mêmes lieux et leur représentation visuelle confortent le sentiment d'enfermement spatial et social. La mise en scène des intérieurs, essentiellement en plans serrés et gros plans, confirme l'absence de liberté de mouvement dont souffre chacun des personnages. À l'issue du générique, un plan serré sur le visage d'Earn renvoie à l'univers carcéral qu'il s'apprête à découvrir dans l'épisode 2. Les draps sont gris, les murs capitonnés sont blanc cassé. D'ailleurs, la palette chromatique d'Atlanta renforce ce parallèle. Même les scènes extérieures sont tournées dans des tonalités froides et métalliques (gris, bleu essentiellement). Au-delà des frontières intérieures, les personnages ne jouissent pas pour autant d'une réelle liberté de mouvement. Les barrières ne sont certes plus aussi explicitement visibles, mais Earn et ses acolytes ne font que buter sur des obstacles invisibles et implicites.

20 Toujours dans l'épisode 1, alors qu'Earn s'entretient avec son cousin, celui-ci interrompt la discussion sous prétexte " qu'il est l'heure ». Circonspect, Earn suit Paper Boi et Darius dans leur rituel quotidien: chaque jour, et à heure fixe, les deux personnages se rendent dans le jardin voisin, font quelques pas, et s'installent dans un 
canapé. Ce plan en extérieur dévoile la pauvreté du quartier qui est occupé par une population à première vue uniforme alors qu'aucune institution n'est présente dans le cadre. Le délabrement des habitations révèle que l'effort de rénovation de la ville à la fin des années 1990 a uniquement profité au cœur de ville ${ }^{26}$. Le nombre de quidams se trouvant dans les rues est significatif d'un taux de chômage élevé. Le rythme quotidien des personnages se met au diapason de l'enfermement spatial. L'espace et le temps se rejoignent donc dans une réalité synonyme de cloisonnement. Le reste de la ville demeure un monde lointain et inconnu, comme le montre le flou de l'arrière-plan dans ces scènes. Mais ce qui intéresse Donald Glover, ce sont justement les frontières entre deux univers étanches. Comme nous allons le voir, ces lieux deviennent des espaces de tension entre différentes classes sociales et raciales.

\section{Conséquences d'un possible dépassement des frontières sociales et spatiales}

21 Le pilote de la série met déjà en scène un espace de tension récurrent et, « de part et d'autre de la frontière, des forces coercitives différentes façonnent, depuis très longtemps parfois, les citoyens ${ }^{27}$ ». Earn se rend à la station de radio locale pour diffuser la chanson Paper Boi. Hiro Murai met en scène un autre parking; là, Earn rencontre Dave, un présentateur blanc et aisé (vague connaissance) pour pouvoir lui donner la « démo ». La scène dans son intégralité témoigne d'un affrontement entre deux univers irréconciliables et met en image une frontière raciale et sociale qui devient physique.

22 Le constat du fossé qui sépare les deux personnages est immédiat. Dave, tout aussi archétypal que Paper Boi, s'extirpe de sa voiture de luxe, smoothies à la main, et, dès sa deuxième réplique, renvoie Earn à sa pauvreté: «Tu sors de cette poubelle?» La hiérarchisation sociale est instantanée et peut expliquer la création d'une nouvelle zone de tension urbaine, car « c'est [...] en milieu urbain [...] que se nouent, s'amplifient et se démultiplient les interactions de tous ordres qui sont au principe de la vie sociale $^{28} \%$. Le parking devient alors un espace symbolique. Souvent lié à la consommation, il incarne lieu de transition frontalier entre deux univers irréconciliables, mais aussi de frustration, du fait du manque de moyen du personnage, faisant de l'argent un facteur d'inégalités.

$\mathrm{Au} v \mathrm{vu}$ des itinéraires des deux protagonistes, les conséquences du rapport de force deviennent explicites. Après un court échange fait d'incompréhensions, l'animateur poursuit son chemin, laissant Earn derrière lui, et pénètre dans la station de radio située dans l'arrière-plan tandis que le travelling avant s'interrompt brutalement. Earn est dans l'obligation de s'arrêter, sa route est coupée par une nouvelle barrière sociale invisible, mais violente. À l'image, il se voit littéralement barrer la route par une benne à ordure dont l'agressivité est symbolique, mais sans équivoque. Il lui est impossible de dépasser ce seuil qui délimite un espace qui lui est socialement interdit. Pourtant, dans sa définition, un seuil doit être compris comme un lieu de passage, et plus encore, il marque « dans l'espace, le temps ou le mouvement une rupture, une discontinuité, une limite ${ }^{29} »$. Une " limite » donc que le personnage ne parvient pas à franchir. Au cours de la première saison d'Atlanta, Donald Glover multiplie les tentatives d'Earn et des autres pour s'extirper d'un milieu social restreint. Les conséquences sont souvent terribles pour les protagonistes qui se voient expressément renvoyés à leur point de 
départ périphérique. En somme, le dépassement de frontière est immanquablement éphémère et synonyme de violences sociales.

La construction du personnage central de la série, Earn, illustre pleinement l'incapacité pour les protagonistes de franchir les barrières socio-spatiales de manière pérenne. Après avoir fréquenté la prestigieuse université de Princeton, Earn est inéluctablement de retour à son point de départ. Transfuge de classe, il n'a pas pu s'adapter à ce nouveau milieu social, éloigné géographiquement et socialement de son environnement. La conséquence de cette « excursion » est encore une fois désastreuse pour lui qui est rejeté par ceux qui ont vécu son départ comme une trahison. Sans foyer fixe, il est désavoué par ses parents, par Van, seul Paper Boi l'accueille, mais avec méfiance. La réaction de ses proches confirme le sentiment de communautarisme propre à chaque « couronne suburbaine».

Earn est un personnage à la construction paradoxale, lui qui se tient entre deux réalités sociales et spatiales. Il refuse de se voir assigner à un espace restreint et est amené à être en perpétuel mouvement. Nombreuses sont les scènes dans lesquelles la caméra suit le personnage dans un plan dorsal alors qu'il erre dans les rues de son quartier. Outre un procédé empathique, de tels plans soulignent l'incapacité pour lui de se situer et de trouver sa place au sein de la ville d'Atlanta. Earn est comme mû par une fuite en avant et devient un protagoniste mobile, sans pour autant savoir dans quelle direction circuler.

La ville est " un mélange inconfortable d'errance et de fixation [en italique dans le texte] ${ }^{30}$ ". et ainsi «la population marginalisée retrouve la condition d'un nomade assigné à résidence $^{31}$ ». Le paradoxe de "nomade assigné à résidence » décrit parfaitement la succession de mouvements erratiques suivie par Earn. Son travail initial renforce d'ailleurs sa condition paradoxale. Il est en effet employé, avant de démissionner, dans un aéroport pour vendre aux voyageurs des assurances. Figé au sol (Earn demeure immobile devant les passagers qui défilent sous ses yeux), il ne peut qu'observer le ballet aérien des avions dans l'arrière-plan. Pourtant, ses tentatives seront nombreuses afin de dépasser les frontières socio-spatiales qui se présentent à lui, notamment au cours de la deuxième saison, et toutes sont liées au milieu créatif du rap.

Un épisode en particulier témoigne de la confrontation de deux univers socialement et racialement antagonistes. Dans Money Bag Shawty (S02E03), Earn hérite d'un billet de cent dollars. Seulement, au vu de la perception qu'ont les «autres » de sa situation sociale, il ne parviendra pas à s'en défaire, souffrant de divers préjugés qui évoluent au gré de sa migration vers le cœur de ville. Earn verbalise l'importance de l'argent comme moyen indispensable pour s'éloigner des périphéries : «J'aimerais bien être de l'autre côté de la barrière ». Au-delà d'un déplacement intra-urbain, la remarque d'Earn soulève un questionnement identitaire intrinsèque. Le personnage va s'efforcer, notamment par son habillement, d'être un « autre » le temps d'une soirée. Délaissant son short et ses t-shirts qui constituent son costume social, Earn opte pour un jean et une veste, telle l'expression de son désir de se fondre dans un espace qui n'est pas le sien.

28 Avant même d'initier son déplacement, la mise en scène contrecarre les ambitions du personnage. Alors qu'il évoque un mouvement en direction du cœur de ville, la verticalité du plan obstrue encore une fois l'horizon et crée une zone de tension. Le décalage paradoxal entre les propos du protagoniste et la mise en scène provoque une certaine ironie dramatique teintée de fatalisme, renvoyant à un déterminisme socio- 
spatial. Même si Earn se donne les moyens - financiers - de quitter le territoire qui lui est assigné, une force qui le dépasse (ici le contrôle du réalisateur qui enferme son personnage dans le cadre) l'empêchera d'accomplir son périple.

L'analyse de la trajectoire d'Earn au cours de la soirée est révélatrice de cette force qui le domine et le renvoie indéfectiblement en marge. Tout commence par une séance de cinéma dans le centre d'Atlanta, où le premier obstacle, social et racial, se dresse devant eux. Ravi de pouvoir s'extraire de son milieu, Earn n'hésite pas à prendre deux places V.I.P. La symbolique est forte puisque le personnage ne supporte plus d'être une entité abstraite et oubliée. Ce simple geste lui donne le sentiment d'occuper une position centrale et donc d'être devenu quelqu'un d'« important ». Ayant le sentiment que "l'argent donne un pouvoir surhumain à celui ou celle qui le possède ${ }^{32}$ ", la liasse de billets dans sa poche lui permet une transgression spatiale. La sensation de pouvoir ne sera que de courte durée et Earn prend immédiatement conscience que son identité périphérique demeure prédominante, malgré l'argent qu'il détient.

L'hôtesse d'accueil refuse le billet de cent dollars dans un geste révélateur de la hiérarchisation sociale qui a cours. La condition financière des deux personnages (Earn et la caissière) n'est pas éloignée, seulement, la dimension raciale prend le pas sur leur précarité. Earn est une première fois victime de son appartenance spatiale, inextricablement liée à son identité africaine-américaine. D'ailleurs, quelques secondes plus tard, un client blanc tend un même billet de cent dollars sans recevoir de rebuffade. Le racisme subi par Earn est confirmé par la demande de la caissière qui exige de voir sa pièce d'identité. Plus qu'un simple document administratif, ce bout de plastique lui interdit toute progression sociale et spatiale, devenant tout le contraire d'un laissez-passer. La scène renvoie à la première frontière rencontrée par le protagoniste lors de son bref entretien avec Dave. La question raciale est au cœur de ces zones de tension et en vient à prédominer sur les considérations sociales.

31 Earn ne parvient toujours pas à franchir le seuil, et la conséquence en est que la ville " allume et ravive des désirs qu'elle ne satisfera pas $^{33}$ ». Le décor du cinéma ne fait que renforcer la frustration d'Earn. Scintillant, dominé par des couleurs vives, le hall à l'image devient le reflet de ses désirs de transgression inassouvis. De plus, aucune ligne de fuite n'est perceptible et le comptoir derrière lequel se retranche la caissière établit la frontière entre " eux et nous ", empêchant toute communication et compréhension. Selon Jean-Christophe Gay «à une échelle plus petite, les conflits raciaux génèrent des formes de privatisation sélective de l'espace ${ }^{34} »$. Le premier segment de l'épisode le confirme, et ce lieu, situé dans le cœur de ville, se voit privatisé selon des critères raciaux qui dominent ceux de classes sociales. Étant l'«autre », le personnage est ressenti comme une menace, un intrus dans un cadre spatial qui n'est pas le sien.

Incapable de dépasser le seuil du hall, afin de pénétrer dans cet espace central, Earn et Van s'excentrent pour se rendre dans un bar situé dans une première couronne suburbaine. S'il pense réussir à se débarrasser de son billet de cent dollars, le personnage est cette fois accusé d'être un faussaire. L'éloignement du noyau urbain explique le fait que le protagoniste soit parvenu à pénétrer dans ce nouvel espace, il n'a cependant pas pu s'y maintenir, avant de regagner définitivement la marge. La soirée s'achève dans un club de strip-tease, plus proche du quartier dans lequel il réside, réussissant cette fois à y rester.

33 La distance qui sépare Earn de son milieu d'origine - les rues défavorisées d'Atlanta correspond à la possibilité pour lui de s'inscrire dans l'espace social. Plus l'éloignement 
est important, plus il est difficile pour lui de franchir les frontières. Au contraire, plus il se rapproche, moins les obstacles rencontrés sont nombreux. En conclusion, sa volonté de transcender son identité s'avère être un échec. Le costume dont il s'est paré au cours de la soirée n'a pas suffi à masquer son origine spatiale et raciale, ainsi que le territoire urbain qui y est rattaché. À plusieurs reprises, Earn est moqué pour son travestissement, notamment par Paper Boi qui exprime une réelle conscience quant à leur condition socio-spatiale : «Des blancs habillés comme toi peuvent avoir un crédit en claquant des doigts, et tu peux même pas dépenser un billet de cent balles". Finalement, cet épisode n'est qu'une succession de frontières que le protagoniste ne parvient pas à franchir. La cartographie de la ville entraperçue dans le générique du pilote semble difficile à déconstruire. Et si dans ces scènes la richesse individuelle est au cœur de ce figement, elle n'est pas le seul facteur d'enfermement comme l'indique l'épisode suivant « Helen » (S02E04).

Van est invitée à participer à un festival célébrant la culture germanique dont elle est originaire. L'épisode, qui place Van au centre du récit, joue avec la confrontation de deux univers, puisque Earn et Van sont les seuls noirs du weekend. Van, unique modèle de stabilité durant la première saison ${ }^{35}$, traverse elle-aussi une crise introspective qui interroge son identité raciale, mais également son identité genrée en tant que femme. Les échanges avec les autres convives lui révèlent qu'elle n'apparaît plus que comme mère, et non pas comme une entité individuelle.

Aux yeux de tous, elle se rapproche du stéréotype de la femme noire, sans travail et qui élève seule son enfant. Selon son amie, la raison en est « qu'elle a choisi le noir ». Par ce mot, Caroline fait référence à Earn, mais le choix du terme laisse à entendre que la décision de Van dépasse la seule identité de son conjoint. Caroline a quant à elle "choisi le blanc», ce qui lui a permis de s'extraire de l'espace urbain dans lequel les deux amies ont évolué enfant, et de s'écarter du modèle sociétal qu'incarne Van à ses yeux. Plus précisément, son statut de mère célibataire semble inquiéter ses interlocuteurs, ou s'agit-il peut-être d'une préoccupation en raison de son éloignement de l'archétype de la famille nucléaire ? Ironiquement, l'épisode s'ouvre sur deux gros plans évocateurs : le premier sur un jouet sans enfant à côté, un autre sur le siège bébé vide dans la voiture. Que devient Van sans son enfant? Quelle est son identité lorsque, le temps d'un weekend, elle n'est pas avec lui?

À deux reprises, et en seulement quelques secondes, elle est présentée comme « la mère de Lottie ", privée de prénom et de nom. Puis, quelques instants plus tard, elle est cette fois introduite en tant que "copine d'Earn". Caroline lui affirme même "qu'elle a besoin de cette identité » qu'elle semble revendiquer, toujours selon elle. Les propos de Caroline expriment un réel désaveu quant à la vie menée par Van. Elle ne voit en son amie que l'archétype de la femme noire des quartiers suburbains. Leurs différents échanges révèlent que Vanessa n'est plus qu'un personnage social « type », vidé de son identité propre. Par le biais des dialogues entre Van et Caroline, Donald Glover dénonce avec une ironie acerbe le fait que «dans les séries télévisées, c'était les pauvres qui parlaient 'noir', marchaient 'noir', qui avaient une attitude 'noire' ${ }^{36} »$. Caroline reproche à Van de ne pas avoir consenti aux efforts nécessaires pour s'éloigner de ce modèle archétypal, à savoir, " avoir choisi le blanc », comme elle a pu le faire.

Avant cela, au cours de l'épisode S01E03, Van questionnait son rôle : " Je suis toujours la 'femme noire furieuse' ", jouant avec les archétypes de son personnage : «C'est moi le cliché ?» dans une réelle mise en abîme. Elle dénonce le préjugé dont sont victimes les 
femmes noires qui sont vues uniquement comme des «Bad Black Mother», convoquant "tout un réseau sémantique qui relie la sexualité débridée à une fécondité non maîtrisée ${ }^{37}$ ». Les stéréotypes dont elles sont affublées dans l'espace médiatique sont indissociables de l'espace social et urbain qu'elles occupent. Il est intéressant de noter l'intersectionnalité des personnages qui sont victimes de préjugés raciaux et sociaux, mais aussi genrés dans le cas de Van. Comme l'analysait Kimberlé Crenshaw, «la violence faite aux femmes est dirigée par d'autres dimensions identitaires telles que la race et la classe ${ }^{38}$ ». Au cours de l'épisode Helen, le personnage fait face à la multiplicité identitaire qui est la sienne, en tant que femme, noire, et appartenant aux classes défavorisées. La succession de préjugés qu'elle doit affronter est révélatrice du large panorama d'exclusions sociales et spatiales qu'elle subit.

Donald Glover multiplie à l'écran les zones de conflits et les barrières (symboliques et matérielles) que les personnages cherchent à dépasser. Dans ce contexte urbain, le terme de conflit doit être compris "non comme des obstacles à l'émergence d'une société idéale et apaisée, mais bien davantage comme des catalyseurs de la constitution de groupes autour de convergences d'intérêts ${ }^{39}$ ".

Chacun d'eux fait le constat objectif du cloisonnement spatial dont il est victime, tout en s'efforçant de s'en extraire. Cependant, leur entreprise se conclut systématiquement sur un échec, les renvoyant à leurs identités. Qu'ils soient considérés comme appartenant aux classes inférieures (d'un point de vue financier), comme africainsaméricains, ou encore comme femme, leurs identités leur interdisent tout rapprochement avec le cœur de ville. Le constat dressé par la série s'avère pessimiste, renforçant le sentiment de déterminisme social et spatial originel :

En un sens, la distribution des activités et des populations dans la ville peut se lire comme l'effet collectif des choix opérés par une multitude d'agents particuliers [...]. Mais, réciproquement, ces données structurelles préexistent aux décisions individuelles dont elles délimitent le champ des possibles ${ }^{40}$.

Pourtant, Donald Glover refuse de sombrer dans le misérabilisme et la victimisation à outrance de ses personnages. Pour cela, il va s'efforcer de répondre à l'enfermement intradiégétique dont ils sont l'objet, notamment par une liberté créatrice et extradiégétique. Cela passe par une mise en lumière de la scène musicale d'Atlanta, le trap, mais également par une hybridité générique qui brise les frontières se dressant face aux protagonistes.

\section{Possibles alternatives à l'enfermement spatial intradiégétique}

41 La création artistique sera au cœur de cette dernière partie, que ce soit le travail de mise en scène, ou la culture musicale essentielle à la narration. Elle devient le moyen privilégié par lequel Glover offre des échappatoires à ses protagonistes, répondant à l'enfermement urbain. Tout d'abord, le sujet de sa série se veut être une objection à l'invisibilisation dont souffrent encore certains groupes de populations minoritaires. En guise d'exemple, en 2018, les personnages féminins représentent $33,1 \%$ des rôles dans les cent films les plus rentables de l'année ; 33 n'ont aucune africaine-américaine, tandis que 89 ne contiennent aucun protagoniste LGBTQI ${ }^{41}$. La multiplication des séries, ainsi que leur popularité croissante, alliée aux différentes options de visionnage et de production (plateformes de vidéos à la demande) offre à voir une diversification 
des personnages principaux. L'univers fictionnel, par son manque de variation identitaire, est cependant encore loin de se faire le reflet du monde extérieur, établissant de fait une norme et une marge.

Glover répond à cette invisibilisation en se concentrant uniquement sur des protagonistes noirs et reléguant les personnages blancs à des seconds rôles ponctuels, en marge du scénario. Par glissement, la périphérie urbaine devient l'espace narratif central. Les choix opérés par Donald Glover rapprochent sa création du cinéma social qui, lui aussi, vise à «faire voir, en rendant visible ce qu'habituellement l'ordre social ne montre pas et ce qu'il préfère cacher ${ }^{42} »$, ce qui prédit une possible association au cinéma horrifique. Et d'ailleurs, comme le remarque Pierre Sansot, «la plupart des villes ont choisi, au contraire, de masquer les déviances, légères ou graves ${ }^{43}$ ", éventuelle explication au renvoi en périphérie, dans la fange, d'une partie de la population définie comme «l'autre».

43 La désinvisibilisation des protagonistes noirs passe par la dimension chorale de la série. Donald Glover n'hésite pas à faire disparaitre son propre personnage d'Earn au profit de Paper Boi, Van, ou encore Darius. La multiplication des portraits s'oppose à l'uniformisation de l'espace mis en scène et révèle une diversité identitaire au sein même d'une géographie fortement délimitée. Outre un effort de désinvisibilisation, Glover entreprend un travail de déconstruction des archétypes et préjugés. Si certains protagonistes apparaissent initialement comme des personnages "types", le fait de projeter la lumière sur eux, le temps d'un épisode ou plus, permet d'en montrer la complexité et de fendre le masque social ${ }^{44}$. De la sorte, Atlanta s'éloigne d'un portrait monolithique et unidimensionnel, réfutant un modèle fréquemment reproché aux séries qui mettent en scène ces quartiers. D'après Catherine Dessinges et Dominique Gendrin : «Ces dernières [les séries] ont souvent été accusées de déformer les espaces urbains à travers une simplification souvent excessive des communautés qui sont représentées et des minorités qui les créent ${ }^{45}$ ».

44 L'identité plurielle des personnages est donc une première stratégie pour contrecarrer l'invisibilisation médiatique et l'enfermement spatial. Mais aux yeux de Donald Glover, le moyen le plus efficace de dépasser les frontières reste l'expression artistique, tant intra qu'extradiégétique. L'existence même de la série transporte ses personnages audelà de l'espace urbain qu'ils occupent. Le créateur de la série tient tout d'abord à mettre au premier plan la vitalité de la scène musicale de la ville d'Atlanta, et plus précisément des quartiers qu'il dépeint. La musique devient le moyen d'expression privilégié d'une jeune génération d'artistes personnifiée par Paper Boi. Plus encore, le rap offre une « cartographie alternative ${ }^{46}$ » des espaces urbains. D'ailleurs, et comme le confirme Murray Forman, le rap occupe une place essentielle dans ces lieux et il est un élément indispensable pour comprendre « la partition spatiale selon les origines » ainsi que ce que représente «être un jeune noir aux États-Unis ${ }^{47}$ ».

45 La bande-son est pratiquement exclusivement composée par des artistes qui occupent l'espace urbain mis en scène; chaque générique permet de faire découvrir au spectateur un nouveau musicien trap. Il s'avère que le personnage de Paper Boi est inspiré de rappeurs locaux, tels que Jeezy ou Gucci Mane, qui, selon le créateur de la série, ne visent pas à glorifier les armes à feu et la drogue, mais posent un constat sur la situation de leur génération à Atlanta ${ }^{48}$. La trame narrative de la série rejoint ainsi la volonté de la scène trap, tout comme elle rend visible cette ville, dans une représentation peu ordinaire. Que ce soit dans Atlanta ou dans les différentes créations 
musicales, l'influence de l'espace urbain se fait clairement ressentir, c'est d'ailleurs une caractéristique de la musique rap selon Murray Forman ${ }^{49}$.

L'expression d'une culture identitaire forte permet à la population locale de s'immiscer dans un espace qui ne veut pourtant pas d'elle. La musique trap dépasse les frontières du quartier et parvient aux oreilles de ceux qui ont pour habitude d'occulter et oublier ses artistes. Si, comme l'affirme Pierre Sansot, « le marginal, loin de dériver, est fixé et presque enfermé dans un lieu déterminé qu'il ne quitte pas si facilement ${ }^{50}$ ", sa culture parvient quant à elle à s'extraire de son lieu de production "déterminé ». La réaction de certain.e.s face à une telle transgression spatiale est parfois violente. L'épisode Money Bag Shawty s'ouvre sur un monologue d'une protagoniste inconnue, blanche, et identifiée comme appartenant à une classe sociale aisée, qui exprime son aversion pour la musique de Paper Boi. Horrifiée par les paroles, elle se résout à les prononcer afin d'en dénoncer le contenu, violent, sexiste et dangereux pour les jeunes générations selon elle. L'avis de cette femme renvoie aux différents stéréotypes liés à la culture rap. Pourtant, la volonté de Donald Glover est bel et bien de dévoiler une autre facette de cette culture.

À l'instar de ses personnages, Donald Glover refuse de se faire enfermer dans une seule et unique catégorie artistique. Il est scénariste (en compagnie de son frère Stephen), metteur en scène, acteur, mais également rappeur (sous le nom de Childish Gambino). Son identité professionnelle multiple a une conséquence directe sur la conception de la série. Donald Glover s'affranchit de nombreuses contraintes et dépasse aisément diverses limites artistiques. Le format sériel est idoine pour une telle entreprise dans la mesure où plusieurs épisodes permettent à Glover de jouer avec les frontières génériques (absurde, horreur, parodique, etc.). L'hybridité dont témoigne Atlanta représente une réponse - créative - à l'enfermement spatial des personnages.

Une fois par saison, Donald Glover rompt toute linéarité narrative et offre un épisode $a$ priori éloigné du propos social de la série. L'épisode «B.A.N » (S01EO7) est une parodie des talk-shows américains, se déroulant sur la chaîne de télévision fictive Black American Network (B.A.N). Paper Boi, invité sur le plateau de l'émission, répond à des sujets politisés et sociaux telles que la brutalité policière, la condition des personnes transgenres ainsi que transraciales, pour autant, le ton parodique prédomine. Les débats fictionnels sont l'occasion de soulever de nombreuses questions identitaires présentes dans d'autres épisodes et dénoncent certains préjugés sur la culture rap. Ainsi, Paper Boi est accusé par le personnage du docteur Debra Holt (spécialiste des réflexions transgenres) de véhiculer des propos sexistes et transophobes dans ses chansons. S'en suit un débat surréaliste et teinté d'absurdité portant sur la population la plus persécutée, les noir.e.s ou les transgenres.

49 Quelques scènes plus tard, un faux reportage brosse le portrait de Harrison, un adolescent noir, persuadé d'être un homme blanc de trente-cinq ans. De manière hautement ironique, l'enquête suit le personnage de Harrison qui est si convaincu d'être blanc qu'il exprime sa lassitude face à ce qu'il considère comme du racisme antiblanc de la part d'autres personnes noires. Afin d'y mettre un terme, il s'apprête à recevoir une opération pour devenir blanc qui lui permettra « d'être de la race qu'il a choisie ». Le ton parodique de l'épisode se double d'une satire concernant les mêmes enjeux spatiaux, sociaux et raciaux que les autres épisodes.

50 Toutefois, «Teddy Perkins » (S02E06) est l'épisode qui atteste réellement d'une rupture scénaristique et d'une hybridité générique. Peu d'humour, si ce n'est grinçant et 
satirique dans cette nouvelle parenthèse narrative, mais de nombreux codes empruntés au genre horrifique. Le personnage d'Earn est absent - en apparence - laissant l'espace intradiégétique à Darius. Après avoir reçu un «tuyau », il se rend au domicile de Teddy Perkins pour acquérir un piano aux touches multicolores. Seulement, son irruption dans la grande demeure de Perkins s'apparente à une excursion dans une maison de l'horreur au cours de laquelle Darius se laisse entraîner toujours plus profondément, devenant le prisonnier du maitre des lieux. Teddy vit seul avec son frère dans l'obscurité la plus complète, conférant une ambiance effrayante et anxiogène. Alors qu'il franchit les grilles de la demeure, dans un grincement angoissant, Darius découvre un grand manoir victorien, archétype des films d'horreur. La suite ne fera que confirmer sa première impression. Teddy Perkins, propriétaire des lieux, est un ancien musicien, au visage blanchi, un whiteface, ou clown blanc triste, hautement ironique. Par ce geste, Donald Glover dénonce le phénomène des «Blackface » qui ressurgissent à l'heure actuelle ${ }^{51}$, à savoir des blancs se grimant en noir, comme au théâtre au XIX siècle et aux origines du cinéma ${ }^{52}$. Il s'agit d'un nouveau processus de réappropriation des codes raciste, un acte répété dans Atlanta.

Glover ne se contente pas de glisser quelques éléments du genre horrifique. Les références sont nombreuses, notamment le nom du personnage, Teddy Perkins, qui évoque l'acteur de Psychose (Psycho, Alfred Hitchcock, 1960), Anthony Perkins. L'apparition de Benny, dans la cave plongée dans l'obscurité, renvoie à la funeste découverte de la mère de Norman Bates dans le film. Mais la référence la plus importante est plus contemporaine: Get Out (Jordan Peele, 2017), dans lequel l'acteur qui incarne Darius, Lakeith Stanfield, apparait. Le film d'horreur de Peele est lui aussi une dénonciation du racisme encore prégnant dans la société américaine. D’ailleurs, son long-métrage suivant Us (2019), autre fable politique horrifique, s'inscrit dans la droite lignée de Get Out en opposant deux familles, une noire, une blanche, dans un jeu de miroir tragique. Comme Peele l'affirme, il cherche, à dénoncer «le racisme, la vénalité et la brutalité de la société étatsunienne ${ }^{53}$ ", le tout dans un effort de désinvibilisation commun à Atlanta.

Outre l'intertextualité horrifique, l'emprunt au genre concerne également la mise en scène. En effet, un plan désaxé archétypal du film d'horreur est notable qui indique une distorsion de la réalité ainsi qu'une menace de l'ordre établi. Par ce plan précis, Glover confirme le lien étroit entre cet épisode et le travail de Jordan Peele, car d'après Eric Dufour, cité par Franck Fischbach, «le cinéma d'horreur donne un visage à une Amérique oubliée $[. . .]^{54}$ ». C'était notamment le cas concernant la production de films d'horreur au cours des années 1970. Des œuvres engagées telles que La colline a des yeux (The Hills Have Eyes, Wes Craven, 1977) ou encore Massacre à la tronçonneuse (The Texas Chain Saw Massacre, Tobe Hooper, 1974) ont été réalisées en réponse à un abandon de certains territoires ruraux au profit d'une urbanisation croissante ${ }^{55}$. La menace à l'ordre établi évoquée par les plans désaxés de Glover et Peele renvoie à la désinvisibilisation de minorités, notamment afro-américaine, dans les productions filmiques et sérielles à l'heure actuelle.

Il ne relève pas du hasard que Glover consacre un épisode entier au cinéma d'horreur (là où les autres hybridités génériques, telles que la parodie, le thriller ${ }^{56}$, etc. sont notables de manière ponctuelle). Le film d'horreur répète toujours une même histoire : le bouleversement d'un ordre social préétabli dû à une menace extérieure. Le possible rapprochement entre "Teddy Perkins » et le cinéma d'horreur, notamment celui de 
Jordan Peele, s'explique par les espaces de rencontres et de conflits analysés plus tôt et qui témoignent d'une opposition entre deux groupes sociaux, entre le " eux et nous " pour consolider le lien avec Us. Plus encore, l'incompréhension notable lors de ces rencontres traduit une "altérité radicale, inassimilable, incompréhensible et même impensable ${ }^{57}$ ».

La réaction déjà évoquée de la mère de famille blanche quand elle écoute la chanson de Paper Boi (S02E03) va dans ce sens et renvoie à la définition du genre horrifique : «[...] on peut parler d'horreur lorsque, dans le monde du réel ou de l'imaginaire, on se trouve en présence de phénomènes qui tendent à susciter chez le spectateur certaines réactions psychiques ou viscérales dans le registre de la peur et/ou du dégoût ${ }^{58}$ ». Le visage de cette femme, filmé dans un gros plan fixe, souligne sa « réaction psychique ou viscérale " face à ce qu'elle considère comme une menace à l'ordre établi. Par son emprunt aux codes de l'horreur, Donald Glover ajoute une tonalité ironique à ses propos, jouant avec les préjugés dont sont victimes ses protagonistes dans une démarche cathartique, autre caractéristique récurrente de ces productions.

Autrement dit, la liberté créatrice de Donald Glover, et plus précisément l'hybridité générique favorisée par le format sériel, répond à l'enfermement spatial des personnages. Par ses ruptures narratives et ses divers emprunts, l'auteur parvient à franchir des frontières pourtant intradiégétiquement hermétiques. Selon JeanChristophe Gay, «l'organisation spatiale du monde est faite de ruptures qui peuvent être nettes ou floues ", ce que mettait en scène Hiro Murai dans le générique du pilote. L'introduction de la ville d'Atlanta consistait en une mosaïque, telle une juxtaposition d'« enclaves " privées de toute communication par le biais de coupes franches. Le format sériel et sa délimitation chronologique et narrative par épisode conforte le sentiment de rupture. Donald Glover s'évertue à atténuer ces ruptures, par l'approche artistique qui est la sienne et qui est faite d'hybridité générique. Les genres sont des instruments de «standardisation et de différenciation » comme l'explique Jacqueline Nacache ${ }^{59}$. Une opposition similaire à la réalité quotidienne des personnages, entre mobilité et figement, est notable dans cette définition. Or, à l'heure actuelle, l'idée de mobilité et de transgression des frontières génériques est souvent défendue, notamment par Rick Altman ${ }^{60}$. Les genres doivent être compris comme des carrefours relationnels entre une multitude de films, une théorie soutenue par Glover et qui prolonge le message socio-spatial véhiculé par sa série. L'hybridité générique, ainsi que la création artistique, devient un moyen d'assouplir une géographie urbaine et sociale rigide qui cantonne ses populations à des espaces confinés.

\section{Conclusion}

Dans Atlanta, Donald Glover se joue des archétypes qu'il inscrit, puis déconstruit d'épisode en épisode. Alors que ses personnages semblent figés dans une identité inextricable du milieu géographique et social qu'ils occupent, le créateur démontre que leurs réflexions identitaires sont considérablement plus complexes. Comme son nom l'indique, la série s'inscrit dans un environnement urbain particulier - le Sud étatsunien - et qui influence directement la construction des protagonistes. Seulement, ils s'efforcent de dépasser le déterminisme de l'enfermement social que Glover dépeint. L'absence d'horizon et d'échappatoire est rendue par la répétition de mêmes lieux, la circularité narrative ainsi que la mise en scène de l'espace. 


\section{BIBLIOGRAPHIE}

ALTMAN, Rick, Film/Genre, Londres, BFI Publishing, 1999.

APTER, David, DE LAVERGNE, Philippe, «Espace public/Espace privé », Politiques et management public, vol. 5, No. 3 (numéro thématique Public, privé : espaces et gestions. Actes du Second colloque international - Lyon - 15/16 décembre 1986 (deuxième partie) - Incertitude des pratiques), 1987, p. 185-203.

BAUDRILLARD, Jean, GUILLAUME, Marc, Figures de l'altérité, Paris, Descartes, 1994.

BREMARD, Bérénice, MICHOT, Julie, SCHMITT, Isabelle, Sur la route... quand le cinéma franchit les frontières, Dijon, Éditions Universitaires de Dijon, 2018.

CHARLTON, Lauretta, « When We're Surrounded by Blackface », New York Times, 9 février 2019, https://www.nytimes.com/2019/02/09/us/race-related-blackface.html.

CRENSHAW Kimberlé, « Mapping the Margins : Intersectionality, Identity Politics, and Violence against Women of Color », in Martha Albertson Finema, Rixanne Mykitiuk (dir.), The Public Nature of Private Violence, New York, Routledge, 1994, p. 93-118. 
DESPONDS, Didier, AUCLAIR, Elizabeth, «Introduction. Les conflits urbains : révélateurs des tensions de la ville en mouvement », in La ville conflictuelle : Oppositions - Tensions - Négociations, éd. Didier Desponds, Elizabeth Auclair, Paris, Le Manuscrit, 2016, p. 41-48.

DESSINGES, Catherine, GENDRIN, Dominique, « La racialisation des espaces urbains à La NouvelleOrléans post-Katrina : étude des représentations fictionnelles dans la série télévisée Treme », Erea, vol. 14, No. 1, 2016, p. 1-33.

FIJALKOW, Yankel, Sociologie des villes, Paris, La Découverte, 2013.

FISCHBACH, Franck, La critique sociale au cinéma, Paris, Vrin, 2012.

FORMAN, Murray, « 'Represent' : race, space and place in rap music », Popular Music, vol. 19, No. 1, 2000, p. 65-90.

GAY, Jean-Christophe, Les discontinuités spatiales, Paris, Économica, 2004.

GOLDBERG, Jacky, « Jordan Peele, réalisateur de 'Us' : ‘Je veux banaliser les visages noirs dans la pop culture' " , Les Inrockuptibles, 19 mars 2019, https://www.lesinrocks.com/2019/03/19/cinema/ cinema/jordan-peele-realisateur-de-us-je-veux-banaliser-les-visages-noirs-dans-la-pop-culture.

GRAFMEYER, Yves, AUTHIER, Jean-Yves, Sociologie urbaine, Paris, Armand Colin, 2015.

HILL COLLINS, Patricia, « 'Get Your Freak On', image de la femme noire dans l'Amérique contemporaine », Volume!, vol. 8, No. 2, 2011, p. 41-63.

HOGGART, Richard, The Uses of Literacy: Aspects of Working-class Life with Special Reference to Publications and Entertainments, Londres, Penguin Books, 1960.

JAUSS, Hans Robert, Pour une esthétique de la réception, Paris, Gallimard, 1978.

LEE, Christina, " What Does Atlanta Hip-Hop Think of 'Atlanta' the Show ? ", The Pitch, 28 février 2018, https://pitchfork.com/thepitch/what-does-atlanta-hip-hop-think-of-atlanta-the-show.

MANZAGOL, Claude, « Une métropole de l'ère globale : Atlanta », Annales de Géographie, No. 591, 1996, p. 516-534.

MARTINET, Marie-Madeleine, CONTE, Francis, VALENTIN, Jean-Marie, MOLINIÉ, Annie, Espaces multiculturels : sociétés et images, Paris, Presse de l'Université Paris-Sorbonne, 2011.

MONNET, Jérôme, Ville et pouvoir en Amérique, Paris, L’Harmattan, 1999.

NACACHE, Jacqueline, Le film hollywoodien classique, Paris, Nathan, 1995.

SANSOT, Pierre, La marginalité urbaine, Paris, Rivages, 2017.

SORLIN, Pierre, «La ville peut-elle être une scène ? Cinéma et urbanisme », Les Annales de la recherche urbaine No. 85 (numéro thématique Paysage en ville), 1999, p. 71-77.

Sitographie :

« 2018 Statistics », Women and Hollywood, https://womenandhollywood.com/resources/statistics/ 2018-statistics/.

\section{NOTES}

1. Georges Molinié, «Introduction à une sémiotique de l'espace ", in Espaces multiculturels : sociétés et images, éd. Marie-Madeleine Martinet, Francis Conte, Jean-Marie Valentin, Annie Molinié, Paris, Presses de l'Université Paris-Sorbonne, 2011, p. 14. 
2. «[...] une série [...] qui entre dans la tradition sérielle de David Simon, qui a pour habitude de mettre en scène d'une manière critique les questions sociales, raciales et politiques dans les grandes villes américaines ». Catherine Dessinges, Dominique Gendrin, «La racialisation des espaces urbains à La Nouvelle-Orléans post-Katrina : étude des représentations fictionnelles dans la série télévisée Treme ", E-rea, Vol. 14, No. 1, 2016, p. 3.

3. Franck Fischbach, La critique sociale au cinéma, Paris, Vrin, 2012, p. 29.

4. L'avènement du Film Noir dans les années 1950 conférait une importance primordiale à l'espace urbain dans lequel était prisonnier le personnage masculin central. Plus éloignés du Film Noir, nous pouvons également penser à Do The Right Thing (Spike Lee, 1988), ou plus récemment, Blindspotting (Carlos Lopez Estrada, 2018), qui mettent en scène un environnement proche de celui d'Atlanta, respectivement à Brooklyn et Oakland.

5. Le nom de Paper Boi est un hommage au rappeur Big Boi qui a participé à l'explosion de la scène trap, courant lié à l'espace urbain d'Atlanta.

6. Yves Grafmeyer, Jean-Yves Authier, Sociologie urbaine, Paris, Armand Colin, 2015, p. 8.

7. David Apter, Philippe de Lavergne, "Espace public/Espace privé », Politiques et management public, Vol. 5, No. 3, 1987, p. 186.

8. Claude Manzagol, "Une métropole de l'ère globale: Atlanta ", Annales de Géographie, No. 591, 1996, p. 516-534.

9. Ibid.

10. Grâce au réalisateur Mathieu Rochet, la chaîne Arte propose une plongée dans l'univers trap. La série Lost in Traplanta (2019), suit le parcours d'un jeune français plongé dans l'univers musical de la ville, à la recherche de ceux qu'il considère comme les fondateurs de la scène trap : Big Boi et André 3000 qui ont lancé le groupe Outkast.

11. Donald Glover joue d'ailleurs de l'archétype qu'il a construit dans un épisode (S01E04) au cours duquel Paper Boi est "traqué » par un influenceur YouTube qui lui rappelle que ce nom existe déjà sous une multitude de déclinaisons, prouvant ainsi qu'il n'est en rien original.

12. Hans Robert Jauss, Pour une esthétique de la réception, Paris, Gallimard, 1978, p. 54.

13. La ville de Los Angeles est frappée par des émeutes à la suite de l'acquittement de quatre policiers blancs accusés d'avoir battu à mort Rodney King, jeune homme noir.

14. Manzagol, p. 529.

15. Grafmeyer, Authier, p. 31.

16. Ibid.

17. Fischbach, p. 45

18. Yankel Fijalkow, Sociologie des villes, Paris, La Découverte, 2013, p. 15.

19. Fischbach, p. 63.

20. Grafmeyer, Authier, p. 32.

21. Richard Hoggart, The Uses of Literacy: Aspects of Working-class Life with Special Reference to Publications and Entertainments, Londres, Penguin, 1960.

22. Grafmeyer, Authier, p. 26.

23. Dessinges, Gendrin, p. 9.

24. Jérôme Monnet, «La ville, le pouvoir, l'Amérique : 'créations humaines, cosmiques comme le soleil, la lune et les étoiles' », in Ville et pouvoir en Amérique, Paris, L'Harmattan, 1999, p. 9.

25. Didier Desponds, Elizabeth Auclair, «Introduction. Les conflits urbains : révélateurs des tensions de la ville en mouvement ", in La ville conflictuelle : Oppositions - Tensions - Négociations, éd. Didier Desponds, Elizabeth Auclair, Paris, Le Manuscrit, 2016, p. 46.

26. Manzagol, op. cit.

27. Jean-Christophe Gay, Les discontinuités spatiales, Paris, Économica, 2004, p. 79.

28. Grafmeyer, Authier, p. 8. 
29. Hélène Brandon, «La route-seuil dans le film noir américain : limites et franchissements », in Sur la route... quand le cinéma franchit les frontières, éd. Bérénice Brémard, Julie Michot, Isabelle Schmitt, Dijon, Éditions Universitaires de Dijon, 2018, p. 39.

30. Pierre Sansot, La marginalité urbaine, Paris, Rivages, 2017, p. 87.

31. Ibid.

32. Fischbach, p. 72.

33. Sansot, p. 80.

34. Gay, p. 39.

35. Van est institutrice, elle est la seule protagoniste à jouir d'une certaine stabilité professionnelle et ses revenus sont obtenus de manière légale.

36. Patricia Hill Collins, "'Get Your Freak On', image de la femme noire dans l'Amérique contemporaine », Volume!, Vol. 8, No. 2, 2011, p. 46.

37. Ibid.

38. Kimberlé Crenshaw, « Mapping the Margins: Intersectionality, Identity Politics, and Violence against Women of Color », in Martha Albertson Finema, Rixanne Mykitiuk (dir.), The Public Nature of Private Violence, New York, Routledge, 1994, p. 93-118.

39. Desponds, Auclair, p. 42.

40. Grafmeyer, Authier p. 48.

41. https://womenandhollywood.com/resources/statistics/2018-statistics/, consulté le 30 octobre 2019.

42. Fischbach, p. 94.

43. Sansot, p. 81.

44. Au cours de l'épisode 2, et après avoir été emmené au commissariat, Paper Boi rencontre des enfants dans son quartier. Ceux-ci jouent à se tirer dessus avec des armes factices, et face à ce spectacle, le rapper initie une leçon de morale sur le danger des armes à feu, sous-entendant que le modèle qu'il représente n'est pas le bon.

45. Dessinges, Gendrin, p. 8.

46. Murray Forman, «'Represent': race, space and place in rap music », Popular Music, Vol. 19, No. 1, 2000, p. 66.

47. Ibid.

48. Christina Lee, "What Does Atlanta Hip-Hop Think of 'Atlanta' the Show?», The Pitch, 28 février 2018, https://pitchfork.com/thepitch/what-does-atlanta-hip-hop-think-of-atlanta-theshow, consulté le 30 octobre 2019.

49. Forman, op. cit.

50. Sansot, p. 90.

51. Lauretta Charlton, «When We're Surrounded by Blackface », New York Times, 9 février 2019, https://www.nytimes.com/2019/02/09/us/race-related-blackface.html, consulté le 12 avril 2019.

52. Donald Glover dénonce également ce phénomène, sous l'identité de Childish Gambino, dans son clip This is America (Hiro Murai, 2018).

53. Jacky Goldberg, « Jordan Peele, réalisateur de 'Us' : 'Je veux banaliser les visages noirs dans la pop culture' ", Les Inrockuptibles, 19 mars 2019, https://www.lesinrocks.com/2019/03/19/cinema/ cinema/jordan-peele-realisateur-de-us-je-veux-banaliser-les-visages-noirs-dans-la-pop-culture, consulté le 12 septembre 2019.

54. Fischbach, p. 95.

55. Le même sujet est au cœur de Délivrance (Deliverance, John Boorman, 1972) qui se déroule en Géorgie (dont la capitale est Atlanta) et qui oppose l'univers urbain à l'espace naturel et sauvage.

56. L'épisode "Woods» (S02E08) se déroule dans un tout autre univers, s'éloignant, temporairement, de l'espace urbain. Après une dispute avec sa petite amie, Paper Boi se perd dans les bois et se fait pourchasser par un vieil homme pour le moins étrange. Dans cet épisode, 
Hiro Murai emprunte les codes du thriller maniant parfaitement les codes de la course poursuite et du suspense.

57. Jean Baudrillard, Marc Guillaume, Figures de l'altérité, Paris, Descartes, 1994, p. 10.

58. Baudrillard, Guillaume, p. 28.

59. Jacqueline Nacache, Le film hollywoodien classique, Paris, Nathan, 1995, p. 19.

60. Rick Altman, Film/Genre, Londres, BFI Publishing, 1999, p. 141.

61. Pierre Sorlin, "La ville peut-elle être une scène ? Cinéma et urbanisme », Les Annales de la recherche urbaine, No. 85, 1999, p. 75.

\section{RÉSUMÉS}

«Le paysage a beau être dégagé et le terrain visible, l'espace cache beaucoup plus de choses qu'il ne révèle ». Donald Glover, artiste polyvalent (musicien, acteur, réalisateur, producteur) illustre ce constat dans la série qu'il dirige, Atlanta (FX, 2016-) et ce dès la séquence introductive du premier épisode. Après une rapide plongée dans le milieu du rap à Atlanta, une succession de plans aériens en plongées verticales « cartographie » la ville d'Atlanta et présente au spectateur plusieurs frontières qui s'avèrent identitaires. L'ouverture de la série dévoile un tissu urbain fait de disparités, entre résidences familiales aisées et quartiers défavorisés où les terrains vagues se multiplient. Les coupes franches entre ces différentes réalités spatiales semblent rendre le dialogue entre elles impossible. Néanmoins, le paysage est " dégagé », mais derrière la verticalité des plans se dissimule une réalité sociale complexe et normée que le showrunner Donald Glover décortique d'épisode en épisode. Après avoir mis en scène les interstices spatiaux qui deviennent des zones de tension, Donald Glover répond aux frontières qui se dressent devant ses personnages par une hybridité générique et une liberté créatrice remarquable lui permettant de s'extirper du cadre, tant formel que sociétal.

Known as a famous musician, actor, director, producer, Donald Glover highlights the fact that an open landscape can conceal various realities and existences. From its opening sequence, Atlanta (FX, 2016-) illustrates the co-existence of different spaces within this particular urban landscape through the use of a series of high-angle shots. The viewer instantly discovers the different identity borders that delimit and track the city of Atlanta. Shots of wealthy familial homes are juxtaposed to other shots of poor suburban areas without any dissolve. The cuts between these different views explicit the impossible communication between the different social groups evolving in these enclaves. In each episode, Donald Glover aims at detailing the social and racial complexity of the urban landscape. After having shot some conflictual spatial interstices, the showrunner oversteps the different borders that his protagonists daily face, through a noticeable generic hybridity and a creative freedom.

\section{INDEX}

Mots-clés : Atlanta, espace, frontière, hybridité, enclaves, carrefours relationnels

Keywords : Atlanta, space, border, frontier, hybridity, enclaves, cultural crossroads 


\section{AUTEUR}

\section{YVELIN DUCOTEY}

Yvelin Ducotey est A.T.E.R à l'Université d'Angers au sein de laquelle il a soutenu sa thèse de doctorat en novembre 2018. Celle-ci s'intitulait : « Le genre biopic : portraits d'hommes et de femmes iconiques ». Angliciste spécialisé en études filmiques, et membre du laboratoire CIRPaLL, il a abordé divers sujet au cours de ces dernières années (représentation féminine dans le biopic, Histoire et Cinéma, le train dans le $7^{\mathrm{e}}$ art, etc.).

Yvelin Ducotey is currently A.T.E.R (teaching assistant) at the University of Angers where he defended his PhD thesis ("The biopic genre : portrayal of iconic men and women") in November 2018. An English teacher specialised in film studies and a member of the CIRPaLL laboratory, he is interested in various academic fields such as gender studies and cinema ; history and cinema, minority representation in films and TV shows. 